Tendências em Matemática Aplicada e Computacional, 3, No. 1 (2002), 173-182.

(C) Uma Publicação da Sociedade Brasileira de Matemática Aplicada e Computacional.

\title{
Dinâmica da Transmissão Vertical do HIV e a Idade Reprodutiva das Adolescentes
}

S.M. RAIMUNDO, Universidade de São Paulo, Faculdade de Medicina, Av. Dr. Arnaldo, 455, 01246-903 São Paulo, SP, Brasil

H.M. YANG, L.L. VENDITE, Universidade Estadual de Campinas, IMECC, DMA, Cx.P. 6065, 13081-970 Campinas, SP, Brasil.

\begin{abstract}
Resumo. Neste trabalho, propomos um modelo matemático para descrever a dinâmica da transmissão vertical do HIV, assumindo que as mulheres suscetíveis tornam-se infectadas pelo HIV a uma taxa constante. O estudo analítico do modelo mostra que, mesmo existindo a transmissão vertical do HIV, a AIDS pode ser mantida na comunidade quando a taxa de natalidade da população e a idade reprodutiva das mulheres satisfazem determinadas condições.
\end{abstract}

\section{Introdução}

De acordo com os dados publicados pela Organização Mundial de Saúde, até a metade do ano de 1991 foram registrados em todo o mundo um total de 371.802 casos de AIDS e o Brasil, com 18.113 casos, foi considerado o terceiro do mundo em número de casos [12]. Desde então, com o objetivo de se entender melhor a dinâmica de transmissão da AIDS, as pesquisas de observações clínicas e epidemiológicas, juntamente com os modelos matemáticos, estão sendo amplamente desenvolvidos [4] [5] [6] [7] [8] [9].

Segundo os dados do Ministério da Saúde, o primeiro caso de AIDS registrado no Brasil foi em 1980. Embora nenhum caso tenha sido registrado entre mulheres até 1983, em 1984 para cada mulher infectada existiam 124 homens; sendo que em 1985 essa razão era 1 mulher para 24 homens; em 1993, 1 mulher para 4 homens; e em 1999/2000, 1 mulher para 2 homens. Em 1985, registrou-se a primeira ocorrência de transmissão vertical.

Uma das causas do aumento da prevalência da AIDS entre mulheres é devido à transmissão do HIV de mães soropositivas para os recém-nascidos, conhecida como transmissão vertical (ou perinatal). Estudos realizados em 1997 na Europa, América do Norte e África mostraram que a taxa de transmissão vertical estava entre 15 a $35 \%$ [11]; no Brasil, desde a primeira metade de 1993, a transmissão vertical já representava $87,9 \%$ de todos os casos de AIDS em crianças [1].

A transmissão vertical do HIV depende da carga viral (viremia) da mãe, que é intensa no início da infecção e na fase final da doença. A gravidez faz baixar as 
células T-CD4 que são as células que participam da defesa imunológica e, consequentemente, a carga viral aumenta. Quanto maior for a carga viral, maior será a probabilidade de transmissão, que pode ocorrer no final da gestação (quando a barreira placentária está muito fina), durante o parto ou na amamentação. Entretanto, é muito difícil medir a transmissão da infecção e o impacto da doença.

Pelo fato da transmissão vertical do HIV ocorrer durante o parto (quando há troca de sangue entre o bebê e a mãe) ou então pela barreira placentária durante a gestação [1], a gestante toma a droga Azitodimidina (AZT). Atualmente, para diminuir a possibilidade da transmissão vertical, a droga AZT é ministrada na gestante a partir da $14^{a}$ semana de gravidez, durante o parto (com uma dose endovenosa) e, regularmente, depois do parto. Após o parto, por um período de 42 dias, o recém nascido também recebe a droga em forma de xarope. Sem a medicação com a droga AZT, a criança infectada apresenta os sintomas da doença com 2 anos de idade, podendo esse prazo chegar até 5 anos; com a medicação, esse prazo pode ser prolongado. Além disso, por não se conhecer ainda os efeitos colaterais, não existem estudos de uso de AZT intra-uterino. O uso de drogas como tratamento para o HIV reduz o risco da transmissão vertical; entretanto, isso não o elimina [2] [3] [10]. Sem o uso de drogas a transmissão vertical ocorre em uma proporção de $25 \%$ e, com o uso da droga, a transmissão diminui para $7 \%$.

Atualmente, observa-se que existe uma redução do risco de transmissão de mãe para filho, mas a quantidade de mulheres infectadas cresce em proporção muito maior. Como consequência, o aspecto social caracterizado pela sexualidade dessas mulheres tem sido considerado um fator relevante no controle da transmissão vertical do HIV. Atualmente, os grupos de pesquisa estão tendo uma preocupação maior sobre questões que envolvem tratamento e prevenção. Como exemplo, citamse fatores relativos à orientação das gestantes portadoras do vírus, e à iniciação sexual das adolescentes. Neste trabalho, e com a mesma preocupação destes grupos de pesquisa, propomos um modelo matemático destacando a idade reprodutiva das adolescentes e a taxa de natalidade da população como questões importantes para a implantação de novas estratégias de prevenção e controle da AIDS em uma comunidade específica. Vale ressaltar também que definimos a idade reprodutiva como sendo a idade na qual as adolescentes iniciam suas atividades sexuais podendo também gerar descendentes.

Com o propósito de discutir tais questões, o modelo proposto descreve então a dinâmica da transmissão vertical do HIV, quando se adota como tratamento, a aplicação de AZT entre gestantes e recém-nascidos soropositivos, levando-se em conta a idade reprodutiva das adolescentes. Na Seção 2, com a suposição básica que as mulheres suscetíveis tornam-se gestantes infectadas pelo HIV a uma taxa constante, formulamos o modelo. Na Seção 3, desenvolvemos a análise de estabilidade do ponto de equilíbrio. Através desta análise e sob o enfoque das questões anteriormente propostas, na Seção 4 apresentamos situações biológicas nas quais a AIDS pode ser controlada na comunidade mesmo ocorrendo a transmissão vertical do HIV. Finalmente, na Seção 5 concluímos que, apesar de sua simplicidade matemática, o modelo mostra que as questões atuais relativas à sexualidade devem ser priorizadas. 


\section{Formulação do modelo}

O modelo determinístico proposto neste trabalho descreve a dinâmica da transmissão vertical do HIV com a população total $N(t)$ dividida em cinco subpopulações: $S(t)$, população de mulheres suscetíveis ao HIV no tempo $t$; $I(t)$, população de gestantes $\mathrm{HIV}^{+}$que recebem tratamento com AZT; $B_{I}(t)$, população de recém-nascidos $\mathrm{HIV}^{+}$do sexo feminino, que recebem tratamento com AZT e são gerados por gestantes $\mathrm{HIV}^{+} ; B_{S}(t)$, população de recém-nascidos do sexo feminino suscetíveis ao HIV que recebem tratamento com AZT e gerados por gestantes $\mathrm{HIV}^{+}$; e $B_{S}^{\prime}(t)$, população de recém-nascidos do sexo feminino suscetíveis ao HIV e gerados por mulheres suscetíveis ao HIV. Dentro deste contexto populacional, assumimos que a população total, $N(t)=S(t)+I(t)+B_{I}(t)+B_{S}(t)+B_{S}^{\prime}(t)$, é suficientemente grande para que cada uma das subpopulações possa ser considerada como uma va-riável contínua; todos os parâmetros do modelo são positivos; existe um fluxo de entrada positivo e constante $(\psi)$ de indivíduos sadios, no compartimento de mu-lheres suscetíveis ao HIV; o modelo com dinâmica vital apresenta um processo de nascimento, com taxa de natalidade $\phi$, e um de morte, com taxas de mortalidade natural e mortalidade pela doença dadas por $\mu$ e $\alpha$, respectivamente; as mulheres suscetíveis ao HIV tornam-se gestantes $\mathrm{HIV}^{+}$a uma taxa constante $r$; e as populações de recém-nascidos do sexo feminino atingem a idade fértil a uma taxa $\sigma$, isto é, $\sigma^{-1}$ é a idade na qual as mulheres atingem a fertilidade.

Os outros parâmetros do modelo são definidos da seguinte forma: $k$, a proporção de filhos do sexo feminino; $p$, a proporção de recém-nascidos $\mathrm{HIV}^{+}$que recebem tratamento com AZT e são gerados pelas gestantes $\mathrm{HIV}^{+} ; q=1-p$, a proporção de recém-nascidos suscetíveis ao HIV gerados pelas gestantes $\mathrm{HIV}^{+} ; \phi k$, a proporção de recém-nascidos do sexo feminino, suscetíveis ao HIV e geradas pelas mulheres suscetíveis; $\phi p k$, a proporção de recém-nascidos $\mathrm{HIV}^{+}$do sexo feminino e filhas de mulheres $\mathrm{HIV}^{+}$; e $\phi q k$, a proporção de recém-nascidos do sexo feminino suscetíveis ao HIV e filhas de mulheres $\mathrm{HIV}^{+}$.

De acordo com as suposições feitas anteriormente, a dinâmica do modelo fica então descrita pelo seguinte sistema não homogêneo de equações diferenciais ordinárias lineares:

$$
\left\{\begin{aligned}
\frac{d S}{d t} & =\psi+\sigma\left(B_{S}^{\prime}+B_{S}\right)-(r+\mu) S \\
\frac{d I}{d t} & =r S-(\mu+\alpha) I \\
\frac{d B_{I}}{d t} & =p \phi k I-(\mu+\alpha) B_{I} \\
\frac{d B_{S}}{d t} & =(1-p) \phi k I-(\sigma+\mu) B_{S} \\
\frac{d B_{S}^{\prime}}{d t} & =\phi k S-(\mu+\sigma) B_{S}^{\prime} .
\end{aligned}\right.
$$

Com o propósito de identificarmos quais parâmetros do modelo que podem contribuir para a prevenção da transmissão vertical do HIV e, conseqüentemente, propiciar um controle mais efetivo da doença na população, a análise matemática do 
sistema (2.1) concentra-se em determinar seu ponto de equilíbrio verificando sua estabilidade.

\section{A estabilidade do equilíbrio endêmico}

O único ponto crítico do sistema não homogêneo (2.1) é o ponto de equilíbrio não trivial, $P^{*}=\left(S^{*}, I^{*}, B_{I}^{*}, B_{S}^{*}, B_{S}^{*}\right)$, dado por:

$$
\left\{\begin{aligned}
S^{*} & =\frac{\psi(\mu+\sigma)}{\mu(\mu+r)\left(1-\frac{\sigma}{\sigma}\right)} \\
I^{*} & =\frac{\psi(\mu+\sigma) r}{\mu(\mu+\alpha)(\mu+r)\left(1-\frac{\sigma}{\sigma_{1}}\right)} \\
B_{I}^{*} & =\frac{p \phi k r}{(\mu+\alpha)^{2}}\left[\frac{\psi(\mu+\sigma)}{\mu(\mu+r)\left(1-\frac{\sigma}{\sigma_{1}}\right)}\right] \\
B_{S}^{*} & =\frac{\psi(1-p) \phi k r}{\mu(\mu+\alpha)(\mu+r)\left(1-\frac{\sigma}{\sigma_{1}}\right)} \\
B_{S}^{\prime *} & =\frac{\psi \phi k}{\mu(\mu+r)\left(1-\frac{\sigma}{\sigma_{1}}\right)},
\end{aligned}\right.
$$

onde definimos:

$$
\sigma_{1}=\frac{\mu \phi_{1}}{\left(\phi-\phi_{1}\right)}
$$

e

$$
\phi_{1}=\frac{(\mu+\alpha)(\mu+r)}{k[\mu+\alpha+r(1-p)]} .
$$

Da definição (3.3), temos que $\phi_{1}>0$, pois $p \in[0,1]$. Além disso, da equação (3.2), podemos observar que se $0<\phi<\phi_{1}$, então $\sigma_{1}<0$. Assim, para o ponto crítico (3.1) ser biologicamente viável (todas as coordenadas positivas) precisamos ter:

$$
\begin{aligned}
& 0<\sigma<\sigma_{1} \quad \text { com } \quad \phi>\phi_{1} \\
& \text { ou } \\
& \sigma_{1}<0 \quad \text { com } \quad \phi<\phi_{1} \text {. }
\end{aligned}
$$

Para determinarmos a natureza da estabilidade do ponto de equilíbrio não trivial (3.1) tomamos as mudanças de variáveis: $S=s+S^{*} ; I=i+I^{*} ; B_{I}=b_{I}+B_{I}^{*}$; $B_{S}=b_{S}+B_{S}^{*}$ e $B_{S}^{\prime}=b_{S}^{\prime}+B_{S}^{\prime *}$, onde $s, i, b_{I}, b_{S}$ e $b_{S}^{\prime}$ são pequenas variações dos valores $S^{*}, I^{*}, B_{I}^{*}, B_{S}^{*}$ e $B_{S}^{\prime *}$, respectivamente. Assim, o sistema não homogêneo (2.1) transforma-se no seguinte sistema homogêneo de equações diferenciais ordinárias 
lineares:

$$
\left\{\begin{aligned}
\frac{d s}{d t} & =\sigma\left(b_{S}^{\prime}+b_{S}\right)-(r+\mu) s, \\
\frac{d b_{S}}{d t} & =(1-p) \phi k i-(\sigma+\mu) b_{S}, \\
\frac{d b_{S}^{\prime}}{d t} & =\phi k s-(\mu+\sigma) b_{S}^{\prime}, \\
\frac{d i}{d t} & =r s-(\mu+\alpha) i, \\
\frac{d b_{I}}{d t} & =p \phi k i-(\mu+\alpha) b_{I} .
\end{aligned}\right.
$$

O sistema (3.5) tem um único ponto de equilíbrio dado pelo trivial, $P_{0}^{*}=$ $\left(s^{*}, b_{S}^{*}, b_{S}^{*}, i^{*}, b_{I}^{*},\right)=(0,0,0,0,0)$. Note que quando $\psi=0, P_{0}^{*}$ também é o ponto de equilíbrio para o sistema (2.1).

A matriz de coeficientes do sistema (3.5):

$$
M=\left[\begin{array}{ccccc}
-(\mu+r) & \sigma & \sigma & 0 & 0 \\
\phi k & -(\mu+\sigma) & 0 & 0 & 0 \\
0 & 0 & -(\mu+\sigma) & (1-p) \phi k & 0 \\
r & 0 & 0 & -(\mu+\alpha) & 0 \\
0 & 0 & 0 & p \phi k & -(\mu+\alpha)
\end{array}\right]
$$

tem cinco autovalores, sendo dois deles facilmente calculados: $\lambda_{1}=-(\mu+\alpha)$; $\lambda_{2}=-(\mu+\sigma)$. Os outros três estão relacionados com o seguinte polinômio característico:

$$
P(\lambda)=-\lambda^{3}-a_{1} \lambda^{2}-a_{2} \lambda-a_{3}=0
$$

onde

$$
\left\{\begin{array}{l}
a_{1}=(\mu+r)+(\mu+\sigma)+(\mu+\alpha), \\
a_{2}=(\mu+r)(\mu+\sigma)+(\mu+\alpha)[(\mu+r)+(\mu+\sigma)]-\sigma \phi k, \\
a_{3}=(\mu+\alpha)(\mu+r)(\mu+\sigma)-\sigma \phi k(\mu+\alpha)-r \sigma \phi k(1-p) .
\end{array}\right.
$$

A análise da estabilidade do equilíbrio trivial pode ser verificada através do critério de Routh-Hurwitz que estabelece quando os autovalores têm parte real negativa. De acordo com este critério, devemos mostrar que se $a_{1}>0, a_{3}>0$ e $a_{1} a_{2}>a_{3}$, então $P_{0}^{*}=(0,0,0,0,0)$ é atrator. Caso contrário, repulsor.

Façamos, primeiro, algumas simplificações. Sejam

$$
\left\{\begin{array}{l}
a_{2}=\{(\mu+r)(\mu+\sigma)+(\mu+\alpha)[(\mu+r)+(\mu+\sigma)]\}\left(1-C_{1}\right), \\
a_{3}=(\mu+\alpha)(\mu+r)(\mu+\sigma)\left(1-C_{2}\right),
\end{array}\right.
$$

com

$$
\begin{gathered}
\left\{\begin{array}{c}
C_{1}=\frac{\phi}{d_{1}} \\
C_{2}=\frac{\phi}{d_{2}}
\end{array}\right. \\
\left\{\begin{aligned}
d_{1}= & \frac{(\mu+r)(\mu+\sigma)+(\mu+\alpha)(2 \mu+r+\sigma)}{\sigma k} \\
d_{2}= & \frac{(\mu+\alpha)(\mu+r)(\mu+\sigma)}{\sigma k[\mu+\alpha+r(1-p)]}
\end{aligned}\right.
\end{gathered}
$$


Por hipótese do modelo todos os parâmetros são positivos e, sendo $0<p<1$, então $C_{1}>0$ e $C_{2}>0$. Além disso:

$$
d_{1}-d_{2}=\frac{[(\mu+r)(\mu+\sigma)+(\mu+\alpha)(2 \mu+r+\sigma)][r(1-p)]+(\mu+\alpha)^{2}(2 \mu+r+\sigma)}{\sigma k[\mu+\alpha+r(1-p)]}>0,
$$

ou seja, $d_{1}>d_{2}$ e da equação (3.9),

$$
C_{1}<C_{2} \text {. }
$$

Com estas simplificações, podemos agora verificar as condições do critério de Routh-Hurwitz:

1. Da equação (3.7), $a_{1}>0$, pois todos os parâmetros são positivos.

2. Da equação (3.8), temos que

$$
a_{3}>0 \Longleftrightarrow 0<C_{2}<1 \text {. }
$$

3. Falta verificarmos $a_{1} a_{2}>a_{3}$. Temos,

$$
\begin{aligned}
a_{1} a_{2}-a_{3}= & (\mu+\alpha)(\mu+r)(\mu+\sigma)\left(C_{2}-C_{1}\right)+(2 \mu+r+\sigma)\left(1-C_{1}\right) \\
& {\left[(\mu+\alpha)^{2}+(\mu+r)(\mu+\sigma)+(\mu+\alpha)(2 \mu+r+\sigma)\right] . }
\end{aligned}
$$

E desde que $C_{1}<C_{2}$, então

$$
a_{1} a_{2}-a_{3}>0 \Longleftrightarrow 0<C_{1}<1
$$

Assim, para as condições (3.12) e (3.13) serem satisfeitas, devemos ter

$$
0<\phi<d_{2}
$$

Observe que quando $d_{2}<\phi<d_{1}$ tem-se $C_{1}<1$ e $C_{2}>1$; quando $\phi>d_{1}$ tem-se $C_{1}>1$ e $C_{2}>1$ e, nesses intervalos, as condições (3.12) e (3.13) não são satisfeitas.

Além disso, substituindo (3.10) em (3.14), temos:

$$
\left\{\phi-\frac{(\mu+\alpha)(\mu+r)}{k[\mu+\alpha+r(1-p)]}\right\} \sigma<\frac{(\mu+\alpha)(\mu+r) \mu}{k[\mu+\alpha+r(1-p)]} .
$$

Definindo

$$
\phi_{1}=\frac{(\mu+\alpha)(\mu+r)}{k[\mu+\alpha+r(1-p)]}>0,
$$

pois $p \in[0,1] \mathrm{e}$

$$
\sigma_{1}=\frac{(\mu+\alpha)(\mu+r) \mu}{\phi k[\mu+\alpha+r(1-p)]-(\mu+\alpha)(\mu+r)}=\frac{\phi_{1} \mu}{\phi-\phi_{1}},
$$


(3.15) será satisfeita quando

$$
\text { ou } \begin{array}{ccc}
0<\sigma<\sigma_{1} & \text { com } \quad \phi>\phi_{1} \\
\sigma_{1}<0 & \text { com } \quad \phi<\phi_{1} .
\end{array}
$$

Portanto, o ponto de equilíbrio trivial $P_{0}^{*}=(0,0,0,0,0)$ será atrator sempre que as condições (3.18) são satisfeitas. Quando uma delas não é satisfeita, $P_{0}^{*}$ é repulsor.

Entretanto, é importante ressaltar que estamos interessados no sistema não homogêneo (2.1), cuja solução completa é a solução geral do sistema homogêneo (3.5) mais uma solução particular do não homogêneo. Assim sendo, generalizamos o resultado anterior, concluindo que o ponto de equilíbrio não trivial (3.1) é atrator se $0<\sigma<\sigma_{1}$ com $\phi>\phi_{1}$ ou $\sigma_{1}<0$ com $\phi<\phi_{1}$. Ou seja, existindo a transmissão vertical do HIV $(0<p<1)$, a AIDS pode ser controlada na comunidade (nível endêmico) somente quando $0<\sigma<\sigma_{1} \operatorname{com} \phi>\phi_{1}$ ou $\sigma_{1}<0 \operatorname{com} \phi<\phi_{1}$.

Além disso, como as condições para o ponto de equilíbrio não trivial (3.1) ser atrator (3.18) são as mesmas para sua viabilidade biológica, (3.4), podemos então enunciar o seguinte resultado: o equilíbrio endêmico (3.1) existe biologicamente se, e somente se, ele é estável.

\section{Discussão}

Inicialmente, vamos definir $\phi_{1}$, o valor limiar da taxa de natalidade para o qual a doença é auto-sustentada na comunidade (nível endêmico) e $\sigma_{1}^{-1}$, o valor limiar da idade na qual as mulheres do grupo de risco (que têm atividade sexual) atingem a idade reprodutiva. Neste contexto, e com a informação de que a transmissão vertical do HIV pode ou não ocorrer durante o parto, as condições (3.18) podem então representar situações biológicas distintas, nas quais a AIDS pode ou não ser mantida na população:

1. Se a taxa de natalidade é alta e maior que o seu valor limiar $\left(\phi>\phi_{1}\right)$, a prevenção da doença na comunidade em nível endêmico pode ocorrer em função do limiar da idade reprodutiva das mulheres. Assim, para a doença se manter na população em nível endêmico é necessário que as mulheres atinjam a idade reprodutiva em idades avançadas $\left(\frac{1}{\sigma}>\frac{1}{\sigma_{1}}\right)$. Em outras palavras, se $\phi>\phi_{1}$ o ponto de equilíbrio não trivial (3.1) é um atrator somente quando $0<\sigma<\sigma_{1}$.

2. Se a taxa de natalidade continua acima do seu valor limiar $\left(\phi>\phi_{1}\right)$, mas a idade reprodutiva das mulheres suscetíveis é menor que o seu valor limiar, $\left(\frac{1}{\sigma}<\frac{1}{\sigma_{1}}\right)$, então não existe um controle da doença. Se as adolescentes que têm vida sexual ativa (grupo de risco) geram descendentes, a AIDS explode na comunidade junto com a população. Em outras palavras, se $\phi>\phi_{1}$ e $0<\sigma_{1}<\sigma$ o ponto de equilíbrio não trivial (3.1) deixa de ser um atrator. 
3. Entretanto, quando a taxa de natalidade é baixa e menor que o seu valor limiar $\left(\phi<\phi_{1}\right)$ e $\sigma_{1}<0$, qualquer que seja o limiar da idade reprodutiva da população das mulheres do grupo de risco, a AIDS é mantida na comunidade em nível endêmico. Em outras palavras, se $\phi<\phi_{1}$ o ponto de equilíbrio não trivial (3.1) é um atrator.

\section{Conclusão}

A proposta deste trabalho foi desenvolver um modelo para descrever a dinâmica da transmissão vertical do HIV quando é aplicado o tratamento entre recém-nascidos e gestantes soropositivos. Através do estudo analítico desse modelo, foi possível concluir que quando a taxa de natalidade da população é baixa, a AIDS pode ser mantida na população em nível endêmico, qualquer que seja a idade reprodutiva das mulheres, ou seja, o controle da doença pode ser mais efetivo mesmo com as adolescentes na idade reprodutiva gerando descendentes. Por outro lado, se a taxa de natalidade da população é alta, a AIDS mantém-se na população em nível endêmico somente quando as mulheres do grupo de risco têm atividade sexual em idade reprodutiva mais avançada. Assim, se as adolescentes na idade reprodutiva geram descendentes, a AIDS explode juntamente com a população.

Como a epidemia da AIDS é um fator preocupante em todo mundo, inúmeros grupos de pesquisa têm surgido com o objetivo de investigarem a qualidade do cuidado dispensado às mulheres soropositivas nos Centros de Referência do tratamento de DST/AIDS. Segundo esses grupos o número de mulheres HIV têm aumentado e indicar os pontos nos quais os serviços de tratamentos à AIDS precisam melhorar para atender às necessidades da população feminina vem sendo um fator determinante no controle da transmissão vertical do HIV. A situação ideal, que é a erradicação, parece agora impossível. No nosso modelo, a AIDS só poderia ser erradicada quando a taxa de infecção fosse $r=0$, e isso reflete a questão social que foi a razão da formulação deste modelo. Estamos distante desta situação ideal, mas alcançar um controle mais efetivo (nível endêmico) da transmissão vertical do HIV ainda pode ser possível, desde que exista um trabalho simultâneo entre tratamento, educação sexual e controle na idade reprodutiva das mulheres.

Finalmente, podemos concluir que para efetivar qualquer medida de controle, não devemos desconsiderar a sexualidade das mulheres. O tratamento aplicado às gestantes e aos recém-nascidos reduz o risco da transmissão vertical, mas pelo estudo analítico do modelo mostramos que, quando associado ao aspecto social e à sexualidade das adolescentes, possibilita novas estratégias de controle e, portanto, um controle muito mais efetivo da doença na população. E, muito embora o modelo tenha sido bastante simples sob o ponto de vista matemático, o resultado epidemiológico mostrou que, mesmo simples, um modelo matemático pode ser um instrumento importante no controle de doenças dentro da comunidade.

\footnotetext{
Abstract. In this paper, we develop a mathematical model to describe the dynamics of the vertical transmission of HIV assuming that the susceptible women become HIV infected at a constant rate. The analytical analysis of the model shows
} 
that even though the vertical transmission of HIV exists, AIDS can just persist or remain endemic in a community when the birthrate and reproductive age satisfy some conditions.

\section{Referências}

[1] E. Amaral, A. Fagundes, N.S.L. Gonçalves et al, Prevalence of HIV and Treponema pallidum infectious in pregnant women in Campinas and their association with socio-demographic factors, São Paulo Medical Journal/RPM, 114, No. 2 (1996), 1108-1116.

[2] E.M. Connor, R.S. Sperling, R. Gelber et al, Reduction of maternal-infant transmission of human immunodeficiency virus type 1 with zidovudine treatment, N. Engl. J. Med., 331 (1994), 1173-1180.

[3] R.E. Dickover, E.M. Garratty, S.A. Herman et al, Identification of levels of maternal HIV-1 RNA associated with risk of perinatal transmission. Effect of maternal zidovudine treatment on viral load, JAMA, 275 (1996), 599-605.

[4] M.C. Elmas, B. Anzen, J.M. Pena, D. Sperandeo, B. Suligoi, J.A.R. Van Den Hoek, P. Vernazza, Incidence and outcome of pregnancies in HIV-infected women, em "12th World AIDS Conference", Geneve, June 28-July 3, 1998.

[5] R. Lutz-Friedrich, B. Buchelolz, D. Klarmann, G. Horneff, G. Notheis, T. Bohler, F. Bergmann, Combining ZVD treatment and elective cesarean section reduces the vertical transmission of HIV-1 below $3 \%$ in the German perinatal cohorts, em "12th World AIDS Conference", Geneve, June 28-July 3, 1998.

[6] L. Mandelbrot, J. Le Chenadec, A. Berrebi, J.L. Bongain, J.F. Benifla, M.J. Delfraissy, Decreased perinatal HIV-1 transmission following elective cesarean delivery with zidovudine treatment, em "12th World AIDS Conference", Geneve, June 28-July 3, 1998.

[7] S.M. Raimundo, "Uma Abordagem Determinística da Interação de Doenças: AIDS e Tb um Presídio", Tese de Doutorado, IMECC, UNICAMP, Campinas, SP, 1996.

[8] S.M. Raimundo, H.M. Yang, R.C. Bassanezi, R.C. Ferreira, The atraction basins and the assessment of the transmission coefficients for HIV and $M$. Tuberculosis infections among women inmates, Journal Biological Sistems, 10 No. 1 (2002), 61-83.

[9] S.M. Raimundo, A.B. Engel, H.M. Yang, R.C. Bassanezi, An approach to estimating the transmission coefficients for AIDS and for tuberculosis using mathematical models, Systems Analysis Modelling Simulation, to appear. 
[10] R.S. Sperling, D.E. Shapiro, R.W. Coombs et al., Maternal viral load, zidovudine treatment, and risk of transmission of human immunodeficiency virus type 1 from mother to infant, N. Engl. J. Med., 335 (1996), 1621-1629.

[11] B.H. Tess, L.C. Rodrigues, M.L. Newell, D.T. Dunn, T.D.G. Lago, The São Paulo Collaborative Study for Vertical Transmission of HIV -1, em " Breastfeeding, genetic, obstretic and other risk factors associated with mother-to-child transmission of HIV -1 in São Paulo State, Brazil", 1997.

[12] World Health Organization-Surveillance, Forecasting And Impact Assesssment Unit (SFI), Update: AIDS cases reported to Global Programme on AIDS, Geneva, July 1991. 